\title{
A Max-Flow Based Approach to the Identification of Protein Complexes Using Protein Interaction and Microarray Data ${ }^{\dagger}$
}

\author{
Jianxing Feng, Rui Jiang*, and Tao Jiang*
}

\begin{abstract}
The emergence of high-throughput technologies leads to abundant protein-protein interaction (PPI) data and microarray gene expression profiles, and provides a great opportunity for the identification of novel protein complexes using computational methods. By combining these two types of data, we propose a novel Graph Fragmentation Algorithm (GFA) for protein complex identification. Adapted from a classical max-flow algorithm for finding the (weighted) densest subgraphs, GFA first finds large (weighted) dense subgraphs in a protein-protein interaction network and then breaks each such subgraph into fragments iteratively by weighting its nodes appropriately in terms of their corresponding log fold changes in the microarray data, until the fragment subgraphs are sufficiently small. Our tests on three widely used protein-protein interaction datasets and comparisons with several latest methods for protein complex identification demonstrate the strong performance of our method in predicting novel protein complexes in terms of its specificity and efficiency. Given the high specificity (or precision) that our method has achieved, we conjecture that our prediction results imply more than 200 novel protein complexes.
\end{abstract}

\section{Index Terms}

Protein complex, protein-protein interaction network, microarray, dense subgraph, maximum network flow, efficient algorithm

Jianxing Feng is with Department of Computer Science and Technology, Tsinghua University, Beijing 100084, China. Email: fengjx06@mails.tsinghua.edu.cn

Rui Jiang is with MOE Key Laboratory of Bioinformatics, Bioinformatics Division TNLIST/Department of Automation, Tsinghua University, Beijing 100084, China. Email: ruijiang@tsinghua.edu.cn

Tao Jiang is with Department of Computer Science, University of California, Riverside, CA 92521. Email: jiang@cs.ucr.edu

* To whom correspondence should be addressed.

$\dagger$ An extended abstract of this paper has been presented at the 7th Annual International Conference on Computational Systems Bioinformatics (CSB), August, 2008, Stanford, CA. 


\section{INTRODUCTION}

With the advances in modern biophysics and biochemistry, it has been widely accepted that the rise of complicated biological functions is largely due to the cooperative effects of multiple genes and/or gene products. This understanding leads to the emergence of high-throughput technologies for identifying interactions between biological molecules and results in the prosperity of interactomics in the post genomics and proteomics era. For example, with the use of yeast two-hybrid assays [1], [2], [3] and pull-down mass spectrometry experiments [4], [5], genome-wide proteinprotein interactions (PPIs) have been identified and encoded into global PPI networks for the model species Saccharomyces cerevisiae (i.e., baker's yeast) [6], [7], [8]. With the improvement of instruments and increase in the throughput, these technologies have also been applied to identify interactions of human proteins, providing an increasing understanding of the global human PPI network [9]. Parallel to the boom of high-throughput identification of PPIs, genomewide microarray experiments regarding the expression of genes across a number of different conditions have also been conducted and resulted in publicly available databases such as the gene expression omnibus [10].

As a major form of the collaborative effects of two or more proteins, protein complexes play important roles in the formation of complicated biological functions such as the transcription of DNA, the translation of mRNA, etc. Traditionally, protein complexes are identified using experimental techniques such as co-immunoprecipitation and mass spectrometry-based approaches, or computational methods such as protein-protein docking based on protein structures. These methods, though successful, can hardly meet the requirement of identifying all protein complexes in known organisms, due to the large number of proteins that exist and the cost of biological experiments. On the other hand, since in most known cases, a protein complex is composed of a group of two or more proteins that are associated by stable protein-protein interactions, computational methods that can make use of abundant data given by the above high-throughput technologies have been demonstrating increasing success [11], [12], [13], [14], [15].

Many studies use PPI data alone for the purpose of identifying protein complexes or biologically functional modules. These methods assume that densely connected components in PPI networks are likely to form functional modules and hence are likely to be protein complexes [16]. Under this assumption, the methods generally use the density of interactions as a main 
criterion and identify protein complexes by finding dense regions in PPI networks. To mention a few, Bader and Hogue [11] proposed a clustering algorithm called MCODE that isolates dense regions in a PPI network by weighting each vertex according to the topological properties of its neighborhood. Andreopoulos et al. [17] presented a layered clustering algorithm that groups proteins by the similarity of their direct neighborhoods. Spirin and Mirny [12] applied three methods (i.e., clique enumeration, super paramagnetic clustering, and Monte Carlo simulation) to an older version of the MIPS PPI network for yeast [7] and produced about 100 dense subgraphs that were predicted to be protein complexes. Their result was found to be superior to many others in terms of the specificity. Pei and Zhang [13] introduced a subgraph quality measure as well as a "seed-refine" algorithm to search for possible protein complexes in a PPI network. King et al. [18] gave a clustering algorithm based on restricted neighborhood search to partition a PPI network into clusters using some cost function. $\mathrm{Bu}$ et al. [19] introduced a spectral method derived from graph theory to uncover hidden topological structures that consist of biologically relevant functional groups. Wang et al. [20] incorporated both a global metric and a local metric to dissect PPI networks. Li et al. [14] found maximal dense regions by merging local cliques according to their affinity. In a subsequent work, Li et al. [15] devised an algorithm, called DECAFF, to address two major issues in current high-throughout PPI data, namely, incompleteness and high data noise.

Another group of methods combine PPI data and microarray gene expression profiles for the purpose of identifying protein complexes or functional modules. For example, Segal et al. combined PPI and microarray data to identify pathways [21]. Chen and Yuan [22] extended a betweenness-based partition algorithm to partition PPI networks with weighted edges using microarray data. Ideker et al. used such a combination to search for regulatory modules and signalling circuits [23]. Guo et al. [24] identified condition-responsive sub-networks in a PPI network by weighting its edges based on gene expression profiles. The program MATISSE presented in [25] combines PPI and microarray data to find tissue/condition specific functional modules. The work was recently followed up in [26] with the introduction of another program, called CEZANNE, that takes advantage of weighted PPI networks whose interactions have been assigned reliable confidence scores.

These methods regard PPIs as static descriptions of the potentially collaborative effects between proteins and treat gene expression profiles as dynamic information of genes under various 
conditions. Since proteins of a complex usually work together to complete certain biological functions, and there exists a simple mapping between genes and the proteins, the combination of PPI and microarray data could clearly help the discovery of protein complexes or functional modules. For the identification of protein complexes, an important question is that to what extent known complexes exhibit co-expression of their members. Jansen et al. investigated this problem on yeast [27]. They found that generally, permanent complexes, such as the ribosome and proteasome, have a particularly strong relationship with expression, while transient ones do not. This result has been confirmed by similar work such as [28] and [29]. In [28], Simonis et al. found that "stable" complexes such as cytoplasmic ribosome and the proteasome are coherently up or down-regulated in many different conditions. However, such coherency is detected at some level in only 71 out of 113 complexes containing at least 5 proteins. These work generally showed that the members of some complexes are correlated in their expressions while the members of the other complexes are not. Therefore, methods for identifying protein complexes that are solely based on expression profiles may not be very reliable.

Besides these methods, there exist some other methods that aim at identifying protein complexes by using comparative interactomics. For example, Sharan et al. [30] identified protein complexes by a comparative analysis of the PPI networks from yeast and bacteria. Hirsh and Sharan [31] developed a probabilistic model for protein complexes that are conserved across two species and applied it to yeast and fly. These methods based on comparative analysis require the availability of quality PPI networks from multiple species and can only identify protein complexes conserved in multiple species. See [32], [33], [34] for more information about aligning and comparing multiple PPI networks.

Despite differences in the approach and the use of data, most of the computational methods mentioned above define the density of a subgraph as the number of its edges over the number of all possible edges in the subgraph and follow a bottom-up local search strategy to find dense subgraphs. For example, Li et al. [15] first found small dense subgraphs (or components) in a PPI network and then merged these components gradually to form protein complex-like subgraphs. Pei and Zhang [13] greedily expanded some carefully selected seed subgraphs until a given criterion was met. Because such a local search strategy in general does not return an optimal solution (with respect to most objective functions), the above bottom-up methods are not guaranteed to find the densest subgraphs in the input PPI network and therefore may miss 
some important protein complexes that are actually dense.

To overcome this drawback, we adopt a new definition of the density of a subgraph (i.e., the number of edges over the number of nodes in the subgraph) and present a top-down method that utilizes the density information in PPI networks explicitly as well as microarray gene expression profiles to identify protein complexes. Our work combines the classic maximum network flow based Densest Subgraph Algorithm (DSA) [35] to find the densest subgraphs with a novel application of microarray data. Our algorithm, named the Graph Fragmentation Algorithm (GFA), first finds dense subgraphs in a PPI network, many of which could potentially be large, and breaks each of them into fragments iteratively by weighting its nodes appropriately in terms of their corresponding log fold changes in the microarray data, until the fragment subgraphs are sufficiently small. An important property of GFA is that it mainly utilizes the protein interaction information so that its performance will not be seriously affected by the possible inconsistency among the expression profiles of the members of a complex.

In order to test the performance of our method, we apply GFA to three widely used yeast PPI networks (i.e., the MIPS, DIP and BioGRID PPI networks) and compare our predictions with the known protein complexes in the MIPS database as well as with those of the latest methods for protein complex identification (that are not based on comparative analysis) given in [23], [11], [36], [25], [37], [15], [26]. The test results clearly demonstrate the superior performance of our method in predicting novel protein complexes in terms of specificity and efficiency (to be defined below). For example, GFA could be tuned to achieve a high specificity of $81 \%$ while maintaining its sensitivity at $71 \%$ on the DIP PPI network. Our method also provides a ranking of the predicted complexes, taking advantage of the multiple conditions (or samples) in the microarray expression data. Predicted complexes with higher ranks are supported by more samples and thus have larger likelihoods to be true protein complexes. Moreover, our predictions result in more than 200 highly ranked dense subgraphs that share little common proteins with the known complexes in MIPS and are thus likely to be novel protein complexes.

\section{MATERIALS AND METHODS}

\section{A. Data sources}

Three PPI datasets concerning Saccharomyces cerevisiae (i.e., baker's yeast) are used. The first one is the MIPS protein-protein interaction network [7], which contains manually compiled 
interactions from the literature and published large-scale experiments. This dataset will simply be denoted as MIPS-PPI. The second one is the DIP protein-protein interaction network [6], denoted as DIP-PPI. The third one is BioGRID protein-protein interaction network [8], which is the most comprehensive one and will be denoted as BioGRID-PPI. The file names, which contains the download dates or release numbers, of the three datasets are PPI_18052006.tab, Scere20081014.txt and BIOGRID_PPI_2.0.35 for MIPS, DIP and BioGRID-PPI, respectively. Because a PPI network is treated as an undirected simple graph, at most one edge will be kept between any pair of proteins. The numbers of nodes (or edges) in the MIPS, DIP and BioGRID PPI networks are 4,554 (or 12,319), 4,947 (or 17,257) and 5,201 (or 71,044), respectively.

We retrieved 51 sets of microarray gene expression data concerning yeast from the GEO database [10] where the log fold changes of expression levels are provided. Each dataset contains multiple samples (or conditions). Totally, 824 samples are contained in the 51 datasets. Since the genes expressed in each sample are different and they could also be different from the genes contained in a PPI network, we will use a sample of the microarray data on a PPI network if it covers at least $90 \%$ of the genes in the network under consideration. For genes that have no expression data in a certain sample, we treat their (log transformed) expression values as 0. Finally, we chose (randomly) 500, 600, and 700 samples to be applied on the MIPS, DIP, and BioGRID PPI networks, respectively.

As in previous studies [11], [12], [14], [15], the MIPS complex database [7] is used as the benchmark (i.e., the truth) to evaluate the protein complexes predicted by our method. ${ }^{1}$ This database contains protein complexes verified manually and those identified by high throughput experiments. We use the manually verified complexes as the benchmark, denoted as MIPSMAN. Furthermore, our algorithm GFA only outputs connected subgraphs, but many complexes in MIPS-MAN are not connected in the above PPI networks. To evaluate our results more reasonably, we decompose each MIPS complex into connected components according to the PPI network under study. We will use MIPS-MAN-COMP to denote the sets of connected complex components obtained from MIPS-MAN. Finally, since GFA mainly focuses on complexes forming dense subgraphs in the PPI networks, it does not output subgraphs consisting of a single node

\footnotetext{
${ }^{1}$ Note that since MIPS complexes are not extracted (computationally) from MIPS-PPI and vise versa, they could serve as a valid benchmark for predicting complexes from MIPS-PPI.
} 
or edge (because they are trivial). Therefore, all complexes or complex components with sizes 1 or 2 are removed from MIPS-MAN-COMP. Note that the actual content of the benchmark MIPS-MAN-COMP depends on the underlying PPI network used. The size of MIPS-MANCOMP is defined as the number of complexes or complex components in the benchmark. On the MIPS, DIP and BioGRID PPI networks, the sizes of MIPS-MAN-COMP are 100, 114 and 134, respectively. ${ }^{2}$ The numbers of complexes and complex components with sizes above 20 , 30 and 40 are 0,1 and 2 in the three MIPS-MAN-COMP benchmarks corresponding to the MIPS, DIP and BioGRID PPI networks, respectively. Besides MIPS-MAN and MIPS-MANCOMP, we will also use the biological process and macromolecular complex categories in Gene Ontology (GO) [38] as benchmarks for additional comparisons with the programs CODEC [37] and CEZANNE [26]. MIPS-MAN and these last two benchmarks from GO were used in [26] to compare methods for finding functional modules in PPI networks.

\section{B. An outline of GFA}

A PPI network is considered as an undirected simple graph, where each node represents a protein, and each edge represents an interaction between two nodes. A common strategy for discovering protein complexes from a given PPI network is to search for dense subgraphs in the network. Many methods based on such a strategy have been proposed in the literature as reviewed in Section I. The density of a subgraph could be defined in several ways. A widely used definition of density is $\delta=2 \cdot|E| /(|V| \cdot(|V|-1))$ [11], [12], where $E$ and $V$ denote the sets of edges and nodes in the subgraph, respectively. A nice property of this definition is that $\delta$ is in $[0,1]$. Another possible definition is $\delta=|E| /|V|$. Both definitions are sensitive to the size (i.e., the number of nodes) of a subgraph. It is easy to see that the first definition favors small subgraphs, as demonstrated in [12], while the second one favors large subgraphs. In fact, when the first definition is applied, we have to add a lower bound on $|V|$ to make the result interesting. We will use the latter definition of density in this work, since there is an elegant algorithm to find the densest subgraph under this definition. Our experimental results will also demonstrate that this definition of density works very well in finding protein complexes.

Theoretically, the problem of finding a subgraph with the greatest density in a graph under

\footnotetext{
${ }^{2}$ These three datasets are provided as online supplementary material.
} 
the first definition is much harder than that under the second one. The problem under the first definition is basically equivalent to finding the largest clique in a graph, a classical NP-hard problem in theoretical computer science [39]. However, there is an elegant and fast algorithm to solve the problem under the second density definition. This algorithm, simply denoted as DSA (i.e., the Densest Subgraph Algorithm), finds a densest subgraph in a graph by iteratively solving a series of maximum flow problems and has the time complexity of $O\left(|E| \cdot|V| \cdot \log \left(|V|^{2} /|E|\right)\right)$ [35]. Although DSA can be iterated to find many dense subgraphs in a PPI network, this approach (alone) will likely not work very well in terms of finding protein complex-like subgraphs, since it tends to find large dense subgraphs while protein complexes are usually small (i.e., containing no more than 20 proteins). Nevertheless, DSA will form the core ingredient of our algorithm GFA for finding protein complexes. GFA actually uses a generalized version of the second density definition: $\delta=|E| / w(V)$, where we assume that the nodes in the graph are weighted (e.g., using the log fold changes in some sample of microarray data) and $w(V)$ denotes the total weight of the nodes in the subgraph. The algorithm DSA mentioned above also works for this generalized definition.

GFA consists of two phases: (1) identify candidate subgraphs from the input PPI network using a single sample of gene expression data, and (2) combine candidate subgraphs from multiple samples to form a ranked list of predicted protein complexes. The basic idea behind the first phase is to iterate DSA to obtain (large) dense subgraphs and then break each large dense subgraph into fragment subgraphs by weighting its nodes appropriately using the log fold changes of the nodes in the sample. This phase is executed on each sample separately. In the second phase, we have to detect and remove redundant (or overlapping) subgraphs found using different samples and rank the subgraphs according to the times that they are found in all samples. The worst case time complexity of GFA, largely determined by the time complexity of phase 1 , is $O\left(|E| \cdot|V|^{2} \cdot \log \left(|V|^{2} /|E|\right) \cdot\right.$ MaxIter $\cdot$ SampleSize $),{ }^{3}$ where the parameter MaxIter limits the number of times that DSA will be iterated to output a single dense subgraph, and SampleSize is the number of samples of the microarray data used in the computation.

\footnotetext{
${ }^{3}$ Note that when each sample is used, at most $|V|$ dense subgraphs could be extracted by GFA. Thus, GFA may iterate DSA $|V|$ times on a sample. Clearly, this is a highly conservative estimation of the time complexity of GFA.
} 


\section{Identification of candidate subgraphs}

Recall that the gene expression data contains several hundred samples. This phase (phase 1) focuses on one sample at a time. For each fixed sample, we denote the log fold change of the expression level of gene $A$ in the sample as $\operatorname{expr}(A)$. As mentioned above, the idea of this phase is to find potentially large dense subgraphs using DSA and then break each large dense subgraph into smaller ones by weighting its nodes appropriately using their corresponding gene expression level information. At the beginning, the nodes in the input PPI network with degree 1 are removed iteratively. (On the MIPS, DIP and BioGRID PPI networks, 35.5\%, 25.5\% and 8.3\% nodes are removed, respectively.) This step reduces the size of the network and will not affect our final result much because a dense subgraph is not expected to contain nodes with degree 1. Then we weight every node uniformly as 1 and run DSA to find a densest subgraph. If the size of the found subgraph is above a certain threshold (denoted as MaxSize), the weight of each node $A$ in the subgraph is multiplied by a factor of $e^{-\operatorname{expr}(A)}$ and DSA is applied again to the subgraph. The effect of this multiplication is that the weights of highly expressed genes in the subgraph are reduced more than those of lowly expressed genes because $e^{-\operatorname{expr}(A)}$ is a decreasing function. The exponential factor of $e^{-\operatorname{expr}(A)}$ in this adjustment was chosen empirically. Note that since DSA maximizes the ratio $|E| / w(V)$, it tends now to find a subgraph with nodes bearing small weights. In other words, the above weighting adjustment favors genes that are highly expressed in the sample. ${ }^{4}$ As an effect, some nodes with large weights may be removed and the subgraph is fragmented. This step is executed iteratively, until either a given maximum iteration count (denoted as MaxIter) is reached or the size of the subgraph is below MaxSize.

Once a sufficiently small dense subgraph is found, all the nodes in the subgraph and all the edges adjacent to any one of the nodes in the subgraph are removed from the PPI network. Then, we remove all the nodes with degree 1 in the remaining network and reiterate the above process of using DSA to find the next sufficiently small dense subgraph. The whole process ends when

\footnotetext{
${ }^{4}$ Note that here we do not use the absolute value of $\operatorname{expr}(A)$ in the multiplying factor because otherwise genes that are more highly expressed than their normal expression levels would be treated in the same way as those that are less expressed than their normal expression levels, and thus such genes could be mixed together in an output subgraph. We think that subgraphs that contain genes whose expression levels change in significantly different ways are unlikely real protein complexes. In fact, we tested the option of using the absolute value of $\operatorname{expr}(A)$ in GFA on a few datasets and found that it always resulted in a slightly worse performance.
} 
the PPI network exhausts. Note that although this step of GFA favors highly expressed genes when it has to break a large dense subgraph returned by DSA into smaller subgraphs, it may very well identify many candidate dense subgraphs consisting of genes with arbitrary expression levels that are not very large (and thus do not have to be broken up).

\section{Combining candidate subgraphs}

The above phase 1 of GFA generates a set of candidate subgraphs for each sample of the microarray data. When all the candidate subgraphs from all the samples are considered, many of them are duplicated or similar. This phase (phase 2) removes duplicated and similar candidate subgraphs and assigns a rank for each dense subgraph that remains. More specifically, duplicates and trivial subgraphs with sizes 1 or 2 are removed and similar subgraphs are merged. However, because of the drastic difference in the densities of the three PPI networks considered in this paper, we have to use two different strategies in this phase. We use a simple strategy for MIPSPPI and DIP-PPI, and a more general (and slightly more complicated) strategy for BioGRID-PPI, because the last network is much denser. We define the overlap score between two subgraph $A$ and $B$ as

$$
\operatorname{overlap}(A, B)=|A \cap B|^{2} /(|A| \cdot|B|) \text {. }
$$

where $|A|$ is the size of the vertex set of subgraph $A$ and $A \cap B$ is the intersection of the two vertex sets of subgraph $A$ and $B$. This overlap score was used in [11], [13], [15]. It is adopted here to formally describe the similarity between two subgraphs. Clearly, the score lies between 0 (if $|A \cap B|=0$ ) and 1 (if $A=B$ ). More importantly, it tends to be small whenever $|A|$ is very different from $|B|$.

1) The simple strategy: The simple strategy simply counts the frequency, i.e., how many duplicates, of each candidate subgraph and ranks the subgraphs by their frequencies. A subgraph with a high frequency is expected to be a promising protein complex (or complex component), since it is dense and many of its nodes are highly expressed in multiple samples. After the frequency of each candidate subgraph is calculated, we check if two candidate subgraphs overlap. If the overlap score between two graphs is above a certain cutoff, denoted as MaxOverlap, they are deemed duplicates and the one with a smaller frequency is simply removed. If the two subgraphs have the same frequency, we arbitrarily remove one of them. 
As shown in our experimental results, this simple strategy works very well on MIPS-PPI and DIP-PPI, mainly due to the sparsity of these networks. For BioGRID-PPI which is much denser, however, the simple strategy does not perform as well as for sparse networks. This is mainly due to the fact that a dense network such as BioGRID-PPI usually contains a large number of dense subgraphs and the sizes of dense subgraphs tend to be large too. Consequently, a large number of microarray samples are needed in order to make the frequencies of dense subgraphs sufficiently high. Therefore, when a limited number of microarray samples are available, the simple strategy could become too conservative since it only counts subgraphs that repeat exactly in all the candidate subgraphs.

2) The more general strategy: As mentioned in the last section, the simple strategy could become too conservative on large dense PPI networks such as BioGRID-PPI. Moreover, when the input PPI network is dense, DSA becomes so slow that we may not afford to examine every sample of the microarray data. Hence, in this case, we need to revise the definition of frequency and introduce a more general strategy to combine results from different samples. Our basic idea here is to merge similar candidate subgraphs (i.e., subgraphs whose overlap scores exceed a certain threshold). Three parameters will be used in this general strategy.

The first one is the sliding window ratio (denoted as $\alpha$ ). This parameter determines the maximum size difference between two subgraphs that will be considered for similarity. Only subgraphs whose sizes differ by less than a factor of $\alpha$ will be compared for similarity. The second one is the parameter MinFrequency (denoted as $\beta$ ), where the frequency of a subgraph is now defined as the number of candidate subgraphs found in the first phase of GFA (on all samples) that are identical or similar to the subgraph (including itself). In other words, it describes the number of samples from which the subgraph is extracted (exactly or approximately). The definition of similarity is given in step 1 of Algorithm 2. This parameter gives a lower bound on the number of mutually similar subgraphs to be merged to form a putative protein complex in the final result. The third one is the support ratio (denoted as $\gamma$ ). When a group of similar subgraphs are merged, a node will appear in the final resultant subgraph if it appears in at least $\gamma$ fraction of the similar subgraphs in the group. Algorithms 1 and 2 below give more details of the merge process.

In Algorithm 1, sliding windows are determined and Algorithm 2 is called on each sliding window. Algorithm 2 calculates the overlap scores of every pair of candidate subgraphs in the 

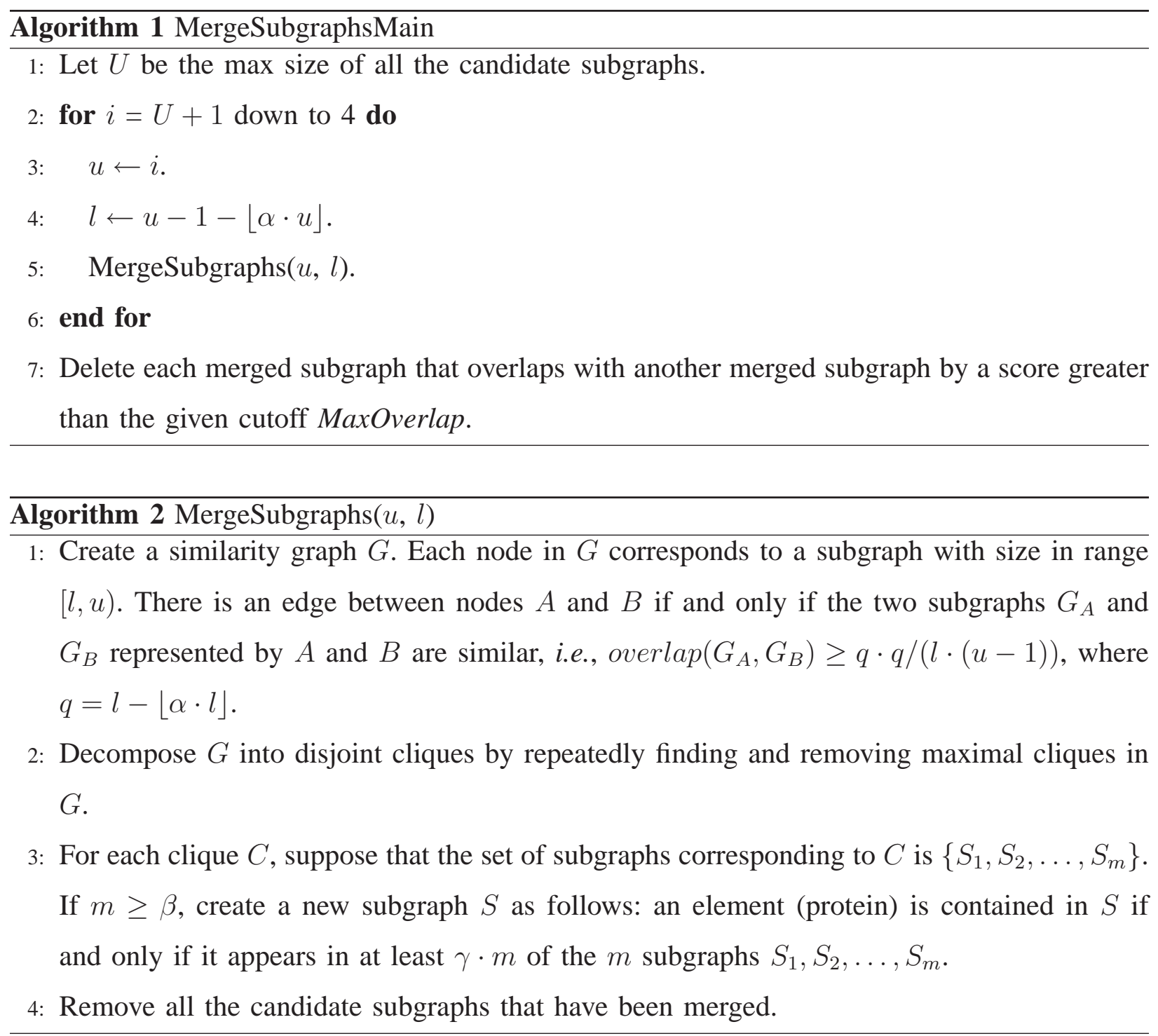

sliding window and merges each group (i.e., clique) of subgraphs that are mutually similar if the group is large enough. Each candidate subgraph being merged is removed so that every subgraph is merged once in the whole process.

The sliding window size $u-l=\lfloor\alpha \cdot u\rfloor$ increases with the size of candidate subgraphs that are processed. We may interpret the parameter $q$ in Algorithm 2 as the minimum number of nodes and edges in a common component among a group of similar subgraphs. When $\alpha=0$ and $\gamma=1$, this general strategy degenerates to the simple strategy.

In the second step of Algorithm 2, a clique with a larger size corresponds to a larger group of dense subgraphs that are going to be merged. Because different dense subgraphs in a clique 
corresponds to different microarray data samples, a clique with a larger size leads to a merged subgraph (as defined at step 3 of Algorithm 2) that is more stable across different conditions (samples) so as to more likely be a true protein complex. However, searching for the maximum clique in the similarity graph $G$ may be infeasible when many samples of the microarray data are combined, because $G$ could be very large and dense in this case. We adopt a common heuristic here: repeatedly find a maximal clique and remove it until the whole similarity graph is completely decomposed. This method is very efficient since it is easy to find a maximal clique.

The parameters involved in the general strategy seem overwhelming. Fortunately, this phase is independent of phase 1, which means that we can run phase 2 of GFA many times with different parameters to obtain a desirable balance between the sensitivity and the specificity. (In the tests presented in this paper, we simply tune the parameters to maximize the sensitivity since our specificity is reasonably high.) Similar to the simple strategy, the candidate subgraphs are ranked by their frequencies. But, there is a subtle difference here. If we run the simple strategy with two MinFrequency values $a$ and $b(a<b)$ to produce two sets of subgraphs $A$ and $B$, then the subgraphs in $B$ are exactly the top $|B|$ subgraphs in $A$. However, the results generated by the general strategy may not have such a "monotonicity" property.

3) A combined strategy: The parameter $\alpha$ is perhaps the most sensitive parameter in GFA. To minimize the influence of $\alpha$, the general strategy is used after the simple strategy. More specifically, the simple strategy is applied to the set of candidate subgraphs $C$ with some given MinFrequency and MaxOverlap, to obtain a group of subgraphs $S$. Every subgraph in $C$ is then compared with those in $S$. If it overlaps with any subgraph in $S$ with a score above MaxOverlap, it is removed from $C$. Then, we apply the general strategy to the reduced candidate subgraph set $C^{\prime}$ and get another set of subgraphs $S^{\prime}$. The final result is the union of $S$ and $S^{\prime}$, where each subgraph that overlaps with another subgraph with a score above MaxOverlap is removed. This two-step strategy is referred to as the combined strategy. Because the simple strategy will remove candidate subgraphs that repeat multiple times exactly, this combined strategy helps the second step of Algorithm 2 by reducing the size of the similarity graph.

\section{E. Default parameters}

In phase 1, MaxSize determines the maximum size of a subgraph found by GFA. In principle, it should be set as the largest possible size of an expected protein complex component (see Section 
II-A for the definition of protein complex components) for a given PPI network. For example, in our experiments, for MIPS-PPI, we select 20 as the bound because the maximum size of a protein complex component in MIPS-MAN-COMP does not exceed 20. Our experiments show that GFA is quite robust with respect to this parameter since its prediction result does not vary much as long as MaxSize is sufficiently large. This is especially true when the microarray data contains many samples, because only the common component of a group of similar subgraphs will be found in multiple samples. For example, we also tried setting MaxSize as 30 on MIPSPPI and observed almost the same result. The parameter MaxIter controls how strictly the size bound is enforced. A small MaxIter may lead to output subgraphs with sizes above MaxSize. This property is useful when there are a few protein complexes that are very dense and much larger than the other protein complexes and we do not want to make MaxSize too large. So, the parameters MaxSize and MaxIter together control the sizes of the output subgraphs. According to our tests, 30 is a sufficiently large value for MaxIter to make sure that the majority (more than $99.75 \%$ ) of the output dense subgraphs have sizes below MaxSize.

For each sliding window, a similarity graph, defined in step 1 of Algorithm 2, will be created. Each clique whose size (i.e., the number of nodes in the clique) above MinFrequency in this similarity graph corresponds to a merged subgraph. The frequency of each merged subgraph is defined as the size of its corresponding clique. When more samples are combined, a sliding window with a certain size may contain more dense subgraphs. Therefore, to use the same MinFrequency, the sliding window size should be reduced by setting a smaller $\alpha$ when more samples are combined. In this case, a larger support ratio $\gamma$ should be used as well. Candidate subgraphs are ranked according to their frequencies. A larger MinFrequency leads to more reliable predictions (see section III). MaxSize, MaxIter and MaxOverlap are independent of the number of samples used but dependent on the PPI network under study. MaxSize should be larger than the largest size of the expected protein complexes. MaxIter should be large enough to make GFA break large subgraphs sufficiently small. MaxOverlap is fixed to 0.2 according to [11], [13], [15].

The values of all the parameters used on the three PPI networks are listed in Table I. Because some of the parameters depend on the number of samples combined, we also list the number of samples used on each PPI network in bold. The table could provide a useful reference point for setting the parameters when different datasets are studied. 
TABLE I

PARAMETER VALUES USED IN OUR EXPERIMENTS

\begin{tabular}{llll}
\hline & MIPS & DIP & BioGRID \\
\hline number of samples & $\mathbf{5 0 0}$ & $\mathbf{6 0 0}$ & $\mathbf{7 0 0}$ \\
MaxSize & 20 & 30 & 40 \\
MaxIter & 30 & 30 & 30 \\
MinFrequency & 3 & 3 & 3 \\
MaxOverlap & 0.2 & 0.2 & 0.2 \\
sliding window ratio & $\backslash$ & $\backslash$ & 0.2 \\
support ratio & $\backslash$ & $\backslash$ & 0.9 \\
\hline
\end{tabular}

The parameters MaxSize and MaxIter are used in phase 1 of GFA. The parameters MinFrequency and MaxOverlap are used in phase 2 by both the simple and general strategies. The parameters sliding window ratio and support ratio are used by the general strategy in phase 2 only.

\section{RESULTS}

\section{A. Matching to the benchmark}

We first introduce some definitions and notations. Since we will validate the predictions by GFA and other methods mainly against the benchmark MIPS-MAN-COMP, we define the effective size of a predicted protein complex as the number of proteins shared by this predicted complex and the complexes in the benchmark. Obviously, we could only hope to validate predicted protein complexes with sufficiently large effective sizes. We say that a protein complex (component) $A$ in the benchmark is identified by a predicted complex $B$ with some cutoff $p$ if

$$
|A \cap B|^{2} /(|A| \cdot|B|) \geq p
$$

A commonly used value for $p$ in the literature is 0.2 [11], [13], [15]. See [11] for an elaborate analysis of this cutoff. We say that $B$ matches $A$ with cutoff $p$ if $A$ is identified by $B$ with the cutoff $p$. To test how this cutoff affects our test results, we also validate the prediction results of different methods using a more stringent cutoff $p=0.5$. The following (shorthand) notations and definitions will be used in the paper:

1) $P$ : The number of predicted protein complexes.

2) $M(p)$ : The number of predicted complexes that match some protein complex component in the relevant benchmark set with cutoff $p$. 
TABLE II

A SUMMARY OF THE PREDICTION RESULT

\begin{tabular}{lllllll}
\hline & $P$ & $I(0.2)$ & $I(0.5)$ & $M(0.2)$ & $M(0.5)$ & $P_{e \geq 2}$ \\
\hline MIPS & 299 & 68 & 42 & 64 & 43 & 72 \\
DIP & 466 & 81 & 52 & 73 & 51 & 90 \\
BioGRID & 574 & 72 & 37 & 73 & 36 & 105 \\
\hline
\end{tabular}

The MinFrequency is fixed to 3 .

3) $P_{e \geq n}$ : The number of predicted protein complexes with effective sizes at least $n$.

4) $I(p)$ : The number of complex components in the relevant benchmark set that have been identified by any one of the predicted complexes with cutoff $p$. This parameter generally reflects the sensitivity of the prediction. As mentioned above, we will consider $p=0.2$ and $p=0.5$ since it could provide more insight into the prediction result.

5) Effective specificity: The number of predicted protein complexes that match complex components in the relevant benchmark set divided by the number of predicted complexes with effective sizes at least 2. In other words, it is equal to $M(p) / P_{e \geq 2}$. Hereafter, the term specificity always refers to effective specificity unless stated otherwise. Note that the notion of specificity as defined here as well as in many PPI network analysis papers (e.g., MCODE,CEZANNE) is often referred to as precision in the information retrieval literature.

6) Sensitivity: The ratio of the complexes in the benchmark that have been identified. It is equal to $I(p) / B$, where $B$ is the size of the benchmark.

7) Efficiency: $I(p) / M(p)$. It describes how many known complexes are covered by a predicted complex that matches some known complexes. Therefore, a highly efficient prediction means that the predicted complexes do not overlap much with each other.

A summary of the prediction results by GFA on MIPS, DIP and BioGRID PPI networks is listed in Tables II and III. ${ }^{5}$ For example, when the cutoffs for identification and matching are both set 0.2 , the sensitivity and the specificity on MIPS-PPI are $68 / 100=0.68$ and $64 / 72=0.89$, respectively. Because of the high efficiency achieved by the prediction results, GFA could predict hundreds of novel protein complexes $\left(P-P_{e \geq 2}\right)$. On MIPS-PPI, for example, GFA predicts $299-72=227$ potentially novel protein complexes.

\footnotetext{
${ }^{5}$ The details of the predictions on the three PPI networks are provided as online supplementary material.
} 
TABLE III

SENSITIVITY AND SPECIFICITY

\begin{tabular}{lllll}
\hline & $\operatorname{Sens}(0.2)$ & $\operatorname{Spec}(0.2)$ & $\operatorname{Sens}(0.5)$ & $\operatorname{Spec}(0.5)$ \\
\hline MIPS & 0.68 & 0.89 & 0.43 & 0.58 \\
DIP & 0.71 & 0.81 & 0.46 & 0.57 \\
BioGRID & 0.54 & 0.70 & 0.28 & 0.34 \\
\hline
\end{tabular}

The sizes of MIPS-MAN-COMP on MIPS-PPI, DIP-PPI and BioGRID-PPI are 100, 114 and 134, respectively. Sens(0.2) and $\operatorname{Spec}(0.2)$ stand for the sensitivity and specificity when cutoff 0.2 is applied, respectively.

We further verify whether proteins in predicted complexes are consistent in their functions by performing a GO analysis. Suppose that in a total of $N$ proteins, $n$ of them share a common GO annotation specified in a certain GO term, while in a predicted protein complex with size $M$, $m$ proteins have the annotation defined by the same GO term, a p-value calculated according to the Fisher's Exact Test as

$$
p=\sum_{k=m}^{\min \{M, n\}} \frac{\left(\begin{array}{l}
n \\
k
\end{array}\right)\left(\begin{array}{l}
N-n \\
M-k
\end{array}\right)}{\left(\begin{array}{l}
N \\
M
\end{array}\right)}
$$

can then be used to characterize the statistical significance of the functional enrichment of the predicted protein complex in the GO term. Note that although more sophisticated and powerful methods exist in the literature [40], [41], [42], [43] for this analysis, the above simple method suffices for us because it confirms that most of the top ranked predicted complexes have good matching GO terms and thus contain proteins that have consistent functions. Because the genes in a protein complex are not expected to have similar molecular mechanisms, the molecular function category in GO is not relevant and thus will not be used in this analysis [25]. We select all the terms under either biological process (GO:0008150) or macromolecular complex (GO:0032991) for this analysis. To focus on GO terms that correspond to specific functions, we collect only GO terms that contain no more than 100 proteins. For each predicted complex, we calculate the above p-value for each of such GO terms and then use the minimal p-value to measure the functional enrichment of the predicted complex. Finally, because we have hundreds of predicted complexes, in order to keep the family-wise error rate not exceeding a preset threshold (e.g., 0.05), we apply the Bonferroni correction [44] to all p-values by multiplying them with the number of predicted complexes and the number of selected GO terms to address the multiple 
hypothesis testing problem. We further vary the maximum number of proteins allowed in a GO term (i.e., 50, 75, 100,125) to assess the impact of this parameter on the analysis, and find that its impact is negligible. For example, when the predicted complexes on MIPS-PPI are matched to GO terms under biological process or under macromolecular complex, the percentages of significant predictions (i.e., those predicted complexes whose corrected p-values for matching with GO terms under either biological process or macromolecular complex are less than 0.05 when) are $33.1 \%$ and $32.8 \%$ when the maximum of number of proteins allowed in a GO term is set to 125 and 50 , respectively.

Table IV shows the top 25 predicted complexes with the highest frequencies. From this table, we can see that many of these putative complexes (e.g., the 1st, 2nd, 3rd, 8th, 10th, 19th and 21th) match complexes in MIPS-MAN-COMP quite accurately. Besides, they are also functionally enriched in some GO terms. For predicted complexes that have no good matches in MIPSMAN-COMP (e.g., the 7th and 14th), they are generally enriched in certain GO terms. For example, the 7th predicted complex contains five proteins (YDR097C, YOL090W, YNL082W, YMR167W, YCR092C). Only three of them (YCR092C, YNL082W and YOL090W) appear in MIPS-MAN-COMP and match the complex (component) 510.180.50.10. However, all the five proteins are members of the "mismatch repair complex" (GO:0032300). In the 14th predicted complex, all the 8 proteins belong to the mRNA cleavage and polyadenylation specificity factor complex (GO:0005847). Due to the incompleteness of data in MIPS-MAN-COMP, only 5 of them appear in MIPS-MAN-COMP and 4 of them match the complex 440.10.20 (pre mRNA3'-end processing factor CFII) in MIPS. The most interesting group of predicted complexes consists of those that have zero effective sizes but have strong GO supports. For example, the 6th predicted complex is totally missed by MIPS-MAN-COMP, but all proteins in this predicted complex are involved in the sphingoid biosynthetic process (GO:0046520). In the 13th predicted complex, 6 out of the 8 proteins are ingredients of preribosome (GO:0030687), a precursor of the eukaryotic cytoplasmic large ribosomal subunit [45]. Finally, the 24th predicted complex matches well with GARP complex (GO:0000938).

\section{B. Comparison with existing methods}

We compare the performance of the proposed GFA algorithm with five existing methods (jActiveModules [23], MCODE [11], MCL [36], DECAFF[15], and MATISSE [25]) on three 
TABLE IV

GO ANALYSIS

\begin{tabular}{|c|c|c|c|c|c|c|c|c|c|c|c|c|c|c|c|}
\hline & $F$ & $M I P S$ & $S$ & $E$ & $B$ & $O$ & $B P$ & $B$ & $O$ & $P$-value & $M C$ & $E$ & $B$ & $O$ & P-value \\
\hline 1 & 447 & $60-1$ & 11 & 11 & 11 & 11 & 0008054 & 12 & 11 & $7.43 e-23$ & 0005680 & 11 & 16 & 11 & $6.06 \mathrm{e}-21$ \\
\hline 2 & 430 & $230.20 .20-1$ & 14 & 14 & 15 & 14 & 0016573 & 41 & 13 & $1.47 \mathrm{e}-17$ & 0046695 & 14 & 19 & 14 & $6.66 \mathrm{e}-27$ \\
\hline 3 & 419 & $440.12 .10-1$ & 5 & 5 & 6 & 5 & 0000292 & 13 & 5 & $3.75 \mathrm{e}-06$ & 0000177 & 5 & 10 & 5 & $6.27 \mathrm{e}-08$ \\
\hline 4 & 400 & $260.90-1$ & 3 & 3 & 6 & 3 & 0000001 & 30 & 3 & $4.42 \mathrm{e}-01$ & 0005885 & 3 & 8 & 3 & $1.36 \mathrm{e}-03$ \\
\hline 5 & 396 & & 3 & 0 & & & 0006470 & 36 & 1 & 1 & & 0 & & & \\
\hline 6 & 394 & & 3 & 0 & & & 0046520 & 4 & 3 & $4.34 \mathrm{e}-04$ & & 0 & & & \\
\hline 7 & 386 & $510.180 .50 .10-1$ & 5 & 3 & 3 & 3 & 0000710 & 9 & 5 & $7.63 \mathrm{e}-08$ & 0032300 & 5 & 6 & 5 & $8.13 \mathrm{e}-10$ \\
\hline 8 & 358 & $133.50-1$ & 3 & 3 & 3 & 3 & 0032784 & 5 & 3 & $1.08 \mathrm{e}-03$ & 0032806 & 3 & 3 & 3 & $2.43 e-05$ \\
\hline 9 & 333 & $320-2$ & 6 & 5 & 4 & 4 & 0034728 & 61 & 6 & $1.11 \mathrm{e}-04$ & 0000788 & 6 & 11 & 4 & $8.46 \mathrm{e}-05$ \\
\hline 10 & 329 & $260.60-1$ & 10 & 10 & 10 & 10 & 0006888 & 88 & 10 & $4.53 \mathrm{e}-09$ & 0030008 & 10 & 10 & 10 & $2.21 \mathrm{e}-22$ \\
\hline 11 & 318 & $500.20 .10-1$ & 4 & 3 & 4 & 3 & 0006409 & 30 & 2 & 1 & 0005853 & 3 & 5 & 3 & $5.76 \mathrm{e}-04$ \\
\hline 12 & 317 & & 3 & 0 & & & 0000002 & 31 & 2 & 1 & 0031942 & 1 & 3 & 1 & 1 \\
\hline 13 & 310 & & 8 & 0 & & & 0042273 & 26 & 4 & $6.39 \mathrm{e}-02$ & 0030687 & 6 & 30 & 6 & $2.78 \mathrm{e}-06$ \\
\hline 14 & 304 & $440.10 .20-1$ & 8 & 5 & 4 & 4 & 0006378 & 18 & 8 & $1.51 \mathrm{e}-12$ & 0005847 & 8 & 15 & 8 & $4.96 \mathrm{e}-14$ \\
\hline 15 & 282 & $290.20 .10-1$ & 6 & 5 & 5 & 5 & 0045039 & 8 & 5 & $8.43 \mathrm{e}-08$ & 0042721 & 6 & 4 & 4 & $2.56 \mathrm{e}-07$ \\
\hline 16 & 281 & $410.20-1$ & 3 & 3 & 4 & 2 & 0006267 & 15 & 3 & $4.94 \mathrm{e}-02$ & 0005656 & 3 & 15 & 3 & $1.11 \mathrm{e}-02$ \\
\hline 17 & 281 & & 3 & 0 & & & 0046839 & 8 & 3 & $6.07 \mathrm{e}-03$ & & 0 & & & \\
\hline 18 & 258 & & 3 & 0 & & & 0051083 & 5 & 2 & 1 & 0005844 & 2 & 17 & 2 & 1 \\
\hline 19 & 249 & $130-1$ & 4 & 4 & 4 & 4 & 0007010 & 61 & 4 & 1 & 0005832 & 4 & 11 & 4 & $1.67 \mathrm{e}-05$ \\
\hline 20 & 242 & & 4 & 0 & & & 0007535 & 5 & 2 & 1 & & 0 & & & \\
\hline 21 & 237 & $510.20-2$ & 3 & 3 & 3 & 3 & 0006360 & 72 & 3 & 1 & 0000120 & 3 & 9 & 3 & $2.04 \mathrm{e}-03$ \\
\hline 22 & 235 & & 4 & 0 & & & 0006097 & 8 & 2 & 1 & & 0 & & & \\
\hline 23 & 229 & & 4 & 0 & & & 0032197 & 95 & 4 & 7.30e-01 & & 0 & & & \\
\hline 24 & 222 & & 3 & 0 & & & 0042147 & 20 & 3 & $1.24 \mathrm{e}-01$ & 0000938 & 3 & 4 & 3 & $9.71 \mathrm{e}-05$ \\
\hline 25 & 218 & & 3 & 0 & & & 0007329 & 5 & 1 & 1 & & 0 & & & \\
\hline
\end{tabular}

The top 25 predicted complexes ranked by GFA on MIPS-PPI. These 25 putative complexes are compared with the benchmark MIPS-MAN-COMP, the biological process category $(B P)$ in GO and the terms rooted under macromolecular complex $(M C)$ in GO. Here, $F$ stands for frequency, $S$ for the size of a predicted complex, $E$ for the effective size, $B$ for the size of the best-matching benchmark complex or GO term, and $O$ for the size of the intersection between a predicted complex and the best-matching benchmark complex or GO term. The column MIPS lists the best matching benchmark complexes. The columns $B P$ and $M C$ list the best-matching GO terms without the prefix "GO:". P-value stands for the adjusted p-values for multiple hypothesis testing with Bonferroni correction. The details of the 25 predicted complexes can be found in the online supplementary material. 18 of them are significantly enriched in either the biological process category or the macromolecular complex category (or both). 
PPI datasets (MIPS, DIP, and BioGRID-PPI). Furthermore, we will compare GFA with two recent programs CODEC and CEZANNE on the PPI datasets considered in the papers that introduced the programs [37], [26]. These methods represent the most advanced methods for predicting protein complexes from PPI and microarray data of a single species.

jActiveModules [23] is one of the first tools that combine PPI and microarray data to find functional modules. In the comparison, except the number of modules returned by jActiveModules, all parameters of $\mathrm{jActiveModules}$ are set to their default values. The number of modules returned by jActiveModules is set to 1000 , the maximum value allowed, because our tests show that this value gives jActiveModules the best performance.

MCODE [11] pioneered the method of identifying protein complexes through finding dense subgraphs in PPI networks. MCL is a clustering algorithm that uses only PPI information to find protein complexes [36]. It was found to be the best performer in predicting protein complexes from PPI data according to the 2006 evaluation study of Brohee and van Helden [46]. A single parameter, inflation, controls the behavior of MCL. In order to tune the performance of the MCL algorithm, we perform a grid search by varying this parameter from 1.2 to 5.9 with step size 0.1 (as suggested in [36]) to obtain the optimal value of this parameter that can give us the highest sensitivity at the identification cutoff $p=0.2$. According to our experience, such an optimal parameter in many cases also leads to reasonably high specificities.

DECAFF [15] is the latest work that uses PPI networks alone to identify protein complexes. Unfortunately, we have only been able to obtain the results of DECAFF on MIPS-PPI and BioGRID-PPI, because the program is not available from the authors. Note that the MIPS-PPI and BioGRID-PPI data used in our studies are the same as those used by DECAFF.

CODEC [37] treats co-immunoprecipitation data as a bipartite graph and identifies dense subgraphs in the bipartite graph. Note that a co-immunoprecipitation dataset could be considered as a PPI network by treating each pray-bait relation as an interaction between the pray and the bait.

MATISSE has five parameters, beta, min seed size, max seed size, min module size, and max module size. In our studies, we set beta to 0.95 as suggested in MATISSE [25], set min seed size to 2 and min module size to 3 for the purpose of predicting protein complexes that contain at least 3 proteins, and set both max seed size and max module size to the default MaxSize value in GFA to make the complexes predicted by MATISSE and GFA have comparable sizes. 
The programs jActiveModules, CODEC, MATISSE and CEZANNE all use microarray data. For each PPI dataset, we apply the same subset of microarray samples from GEO used by GFA to MATISSE. Since we will compare GFA with CODEC and CEZANNE on the datasets in [37], [26], we will use the reported prediction results of CODEC and CEZANNE instead of running these programs. jActiveModules requires p-values as computed by another program VERA [47] for each protein in the input PPI network. However, we were unable to make VERA work for the GEO microarray dataset or the dataset in [26] because each of them is missing some information required by VERA. Therefore, we use the microarray dataset studied in [47] that contains the p-values generated by VERA as a part of the input to jActiveModules. Note that the programs jActiveModules, MATISSE and CEZANNE were not designed to search for protein complexes specifically.

In addition to these existing methods, we also run GFA with all gene expression values randomly generated from a uniform $(-1,1)$ distribution. We call this method $G F A-R$ and use it to check the effect of microarray data on the performance of GFA. In our studies, we set MinFrequency to 3 for both GFA and GFA-R to ensure highly confident predictions.

In the first group of comparisons, the prediction results of GFA, GFA-R, MCODE, MCL, DECAFF, and MATISSE on MIPS, DIP and BioGRID-PPI are compared with benchmark MIPS-MAN-COMP. The performance of each method is evaluated in terms of sensitivity and specificity. All predicted complexes with fewer than 3 proteins are removed before the calculation of sensitivity and specificity, because the benchmark contains only complex components of sizes at least 3. The comparison results are shown in Figure 1. The same set of methods are also compared using benchmark MIPS-MAN (i.e., the manually curated MIPS complexes that are not decomposed into connected components) and $F$-measure which was used recently in [26] to compare various functional module prediction methods. The F-measure is defined as $F=2 *$ sensitivity $*$ specificity/(sensitivity + specificity). Here, a predicted complex is said to match a benchmark complex if the corrected p-value (as described in the above GO analysis section) of matching the two subsets of the proteins contained in the complexes is less than 0.05. ${ }^{6}$ Again, a predicted complex is a true positive if it matches some benchmark complex and a benchmark complex is identified if it has a matching predicted complex. As before, only

\footnotetext{
${ }^{6}$ Note that a cutoff of 0.0001 on uncorrected p-values was used in [26].
} 
predicted complexes of effective sizes at least 2 are considered in the calculation of F-measure. The comparison is shown in Figure 2.

Figure 1 shows that GFA outperforms not only methods using PPI data alone (i.e., MCL, MCODE and DECAFF), but also MATISSE and jActiveModules which use both PPI and microarray data in specificity. This superiority is more obvious when the identification cutoff is set to $p=0.5$. GFA also outperforms MCL, MCODE, MATISSE, and jActiveModules in sensitivity. ${ }^{7}$

DECAFF achieves the highest sensitivity, while GFA achieves the highest specificity. The high sensitivity of DECAFF is mainly due to its large number of predictions. For example, DECAFF predicts 2,840 complexes on BioGRID-PPI, and many of them highly overlap with each other. GFA, on the other hand, predicts only 574 complexes. Although MCL was reported as the best among protein complex prediction methods in a previous study [46], the results in Figure 1 demonstrate that GFA outperforms MCL in terms of both sensitivity and specificity. When F-measure is used (as shown in Figure 2), GFA outperforms MATISSE, MCODE and jActiveModules on all three PPI networks. GFA also outperforms MCL on MIPS-PPI and DIP-PPI and has a comparable performance as MCL on BioGRID-PPI. Compared with GFA, DECAFF performs slightly better on MIPS-PPI but significantly better on BioGRID-PPI. As stated before, the reason that DECAFF achieves better F-measures on MIPS and BioGRID PPI networks is largely due to its large numbers of predicted complexes that overlap with each other significantly. Indeed, if we remove overlapping complexes output by DECAFF using the same procedure in phase 2 of GFA, the F-measures of DECAFF drop from $41.8 \%$ and $57.8 \%$ to $31.5 \%$

\footnotetext{
${ }^{7}$ We estimated the statistical significance of the improved performance of GFA over MCL, DECAFF, MATISSE, MCODE and jActiveModules on MIPS-PPI and DIP-PPI when the identification cutoff $p$ is set to 0.5 using a standard Pearson $\chi^{2}$ test with Yates' continuity correction. The results are as follows. On MIPS-PPI, the sensitivity (43/100) of GFA is better than that of MCL (30/100), MATISSE (13/100), MCODE (25/100) and jActiveModules (4/100) with p-values of 0.04, 2.47e-6, 5.58e-3 and 1.86e10, respectively, and the specificity (42/72) of GFA is better than that of MCL (30/77), DECAFF (152/757), MATISSE (13/70), MCODE (25/48) and jActiveModules (6/562) with p-values of 0.01, 3.47e-13, 1.36e-6, 0.31 and 1.53e-65, respectively. On DIPPPI, the sensitivity (52/114) of GFA is better than that of MCL (25/114), MATISSE (6/114), MCODE (12/114), jActiveModules (0/114) with p-values of 1.36e-4, 3.88e-12, 4.51e-9 and 4.15e-16, respectively, and the specificity (51/90) of GFA is better than that of MCL (24/95), MATISSE (6/68), MCODE (12/38) and jActiveModules (0/894) with p-values 1.34e-5, 8.04e-10, 8.19e-3 and 5.14e-116, respectively. Therefore, except the improvement over MCODE's specificity on MIPS-PPI, all other improvements are statistically significant.
} 
and $37.5 \%$ on the two PPI networks, respectively.

The positive effect of gene expression profiles on the identification of protein complexes is also clearly demonstrated in the comparison between GFA and GFA-R. In terms of the specificity, GFA outperforms GFA-R on all datasets. In terms of the sensitivity, GFA outperforms GFA-R on all datasets except MIPS-PPI. When the identification cutoff is set to $p=0.2$, GFA-R has a slightly better sensitivity over GFA. The main reason behind this is that the predictions of GFA are concentrated on high frequencies. Consequently, GFA outputs much fewer predictions than GFA-R. For example, when MinFrequency is set to 3 and the identification cutoff is set to $p=0.2$, GFA only predicts 299 complexes, while GFA-R predicts 401 complexes on MIPS-PPI. Because the only difference between GFA and GFA-R is that GFA uses microarray data while GFA-R uses random expression profiles, the superior performance of GFA suggests that gene expression profiles do have positive contributions to the prediction of protein complexes. On the other hand, it is interesting to notice that GFA-R performs better than jActiveModules even though the latter uses microarray data.

In the second group of comparisons, we compare GFA with CODEC and CEZANNE in terms of F-measure on three benchmarks including MIPS-MAN, GO:biological process, and GO:macromolecular complex, as done in [26]. To compare with CODEC, GFA is run on the PPI network obtained from the co-immunoprecipitation data given in [5] (which was used in [37]) and the same set of microarray data that we used above for running GFA on MIPS-PPI (i.e., the microarray data with 500 samples). All the parameters of GFA are set to the same values as above when GFA was run on MIPS-PPI. The prediction of GFA is compared with the results reported by CODEC using the $w_{1}$ scoring scheme, which gives CODEC the best performance. To compare with CEZANNE, the same PPI network from [48] and microarray data [49] considered in [26] are used to construct the input for GFA. All edges with confidence scores below 0.2 (one of the cutoffs considered in [48]) are removed and the edge weights (i.e., confidence scores) of the PPI network are then ignored. Again, all the parameters of GFA are set to the same values as above when GFA is run on MIPS-PPI. The comparisons, as depicted in Figure 3, show that GFA in general performs better than both CODEC and CEZANNE on the two datasets. The only exception is that when GO:macromolecular complex is used as the benchmark, CODEC performs slightly better than GFA. The low F-measures of CEZANNE are due to its low sensitivities. Because the aim of CEZANNE is to identify tissue/condition 

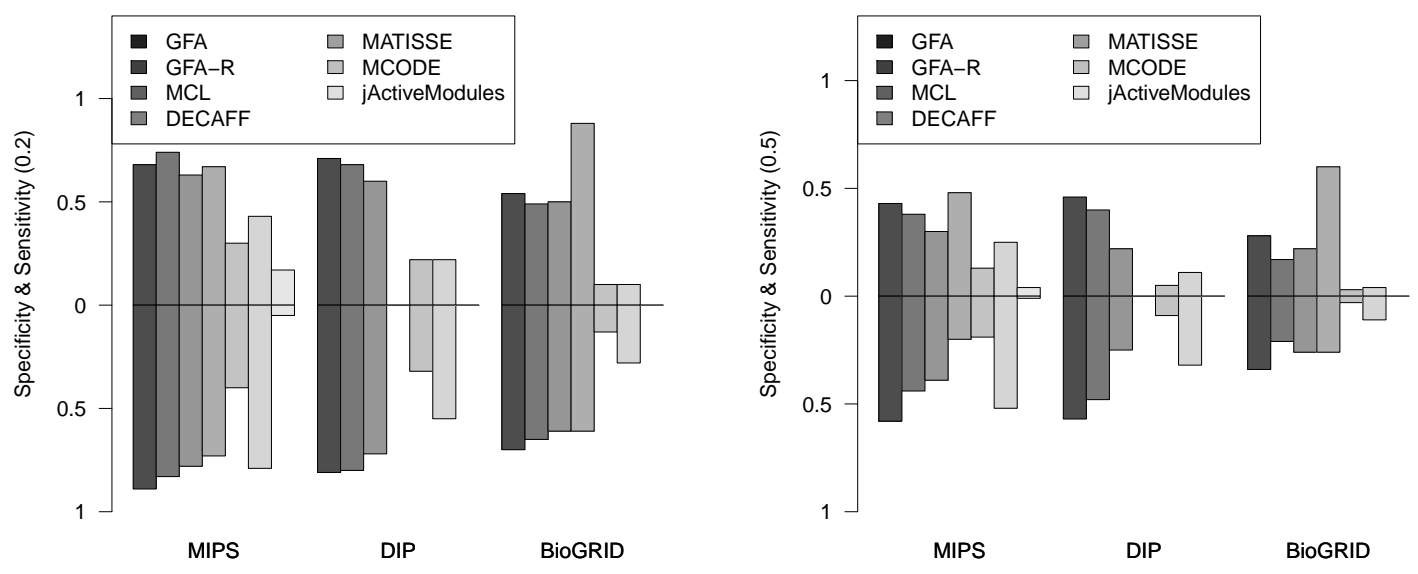

Fig. 1. Comparison of the sensitivities and specificities of the predictions of GFA, GFA-R, MCODE, MCL, DECAFF, MATISSE, and jActiveModules on MIPS, DIP and BioGRID-PPI using MIPS-MAN-COMP as the benchmark. The cutoffs for the identification are 0.2 (left) and 0.5 (right). The sensitivities and specificities obtained simultaneously by different methods are shown in the upper and lower half planes, respectively.

specific functional modules, it predicted only 14 complexes/modules, which led to very low sensitivities (e.g., 14.1\% on GO:macromolecular complex) but high specificities (e.g., 84.6\% on GO:macromolecular complex).

\section{Ranking by frequency}

GFA ranks predictions according to frequencies of the found dense subgraphs. A subgraph with a higher frequency will be ranked higher in the list of candidates. It is therefore necessary to verify whether subgraphs with higher frequencies are more likely to correspond to true protein complexes. For this purpose, we collect subgraphs whose frequencies are above a certain threshold and calculate the fraction of these subgraphs that have matches in the benchmark. This should provide equivalent information as setting different MinFrequency thresholds and observing how the specificity changes with the threshold values. As shown in Figure 4, the specificity decreases with the decrease of MinFrequency, suggesting that candidate subgraphs with higher frequencies match the benchmark better. This observation is more obvious when the identification cutoff is set to $p=0.5$. 


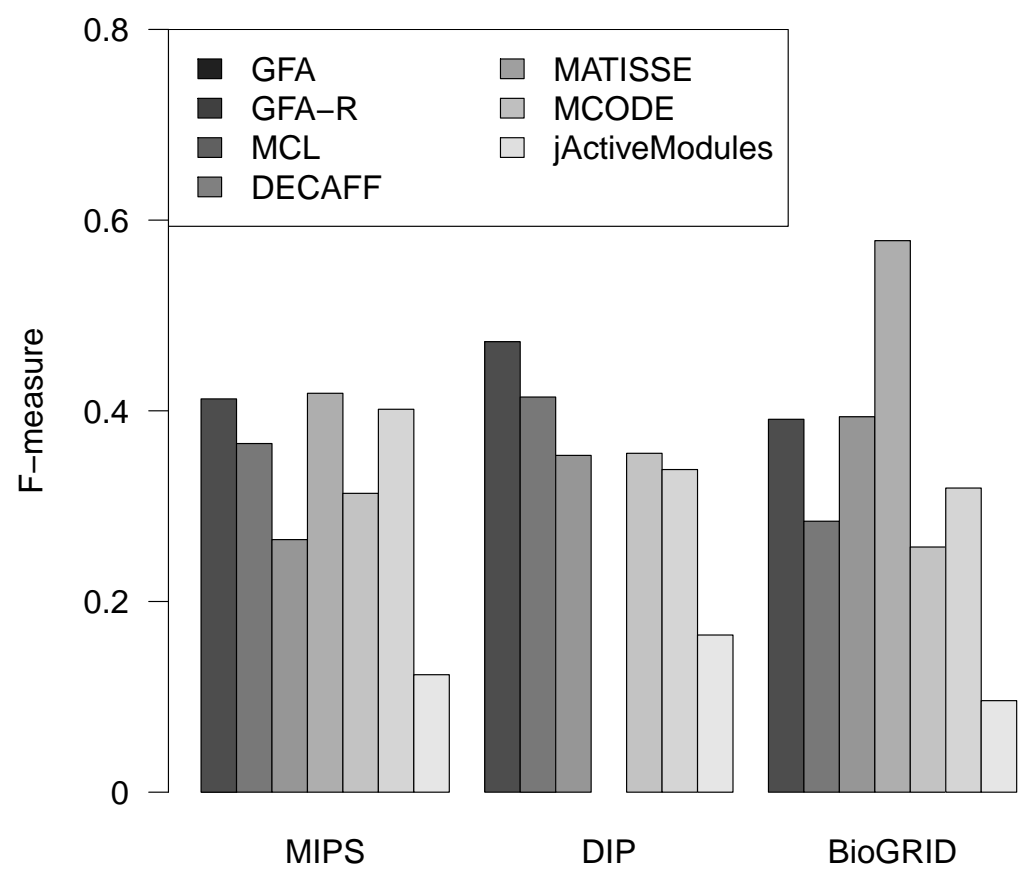

Fig. 2. Comparison of GFA, GFA-R, MCODE, MCL, DECAFF, MATISSE, and jActiveModules on MIPS, DIP and BioGRIDPPI using F-measure and benchmark MIPS-MAN.

We further verify if the proteins in more highly ranked subgraphs are more consistent in their functions by performing the same GO analysis that is used in section III-A. Fixing MinFrequency $=3$, we rank the predicted protein complexes in a non-decreasing order according to their frequencies (i.e., a high frequency corresponds to a small rank). At each rank cutoff, we collect the predictions that are ranked higher than the cutoff, count the number of predictions that are functionally enriched at a certain threshold (e.g., their corrected p-values for matching with terms under either biological process or macromolecular complex categories are less than 0.05), and calculate the ratio (fraction) of the significant predictions. By doing this, we obtain a curve that describes how the ratio of functionally enriched predictions changes over different ranks, as shown in Figure 5.

From the figure, we can see that the ratio of significant predictions is in general high when the 

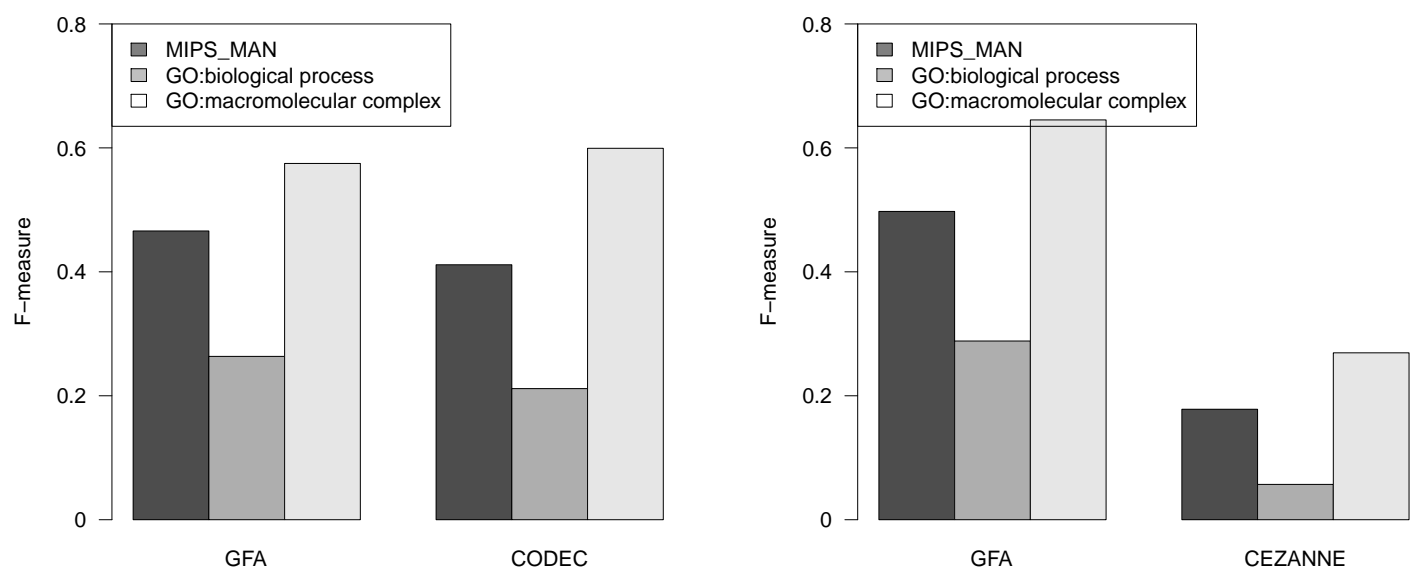

Fig. 3. Comparison of GFA with CODEC and CEZANNE in terms of F-measure on the datasets used in [37] and [26], respectively. The prediction results of CODEC and CEZANNE are downloaded from the websites given in [37], [26].

rank cutoff is small and low when the rank cutoff is large, indicating that more highly ranked predictions are more likely to be enriched in a certain GO term. Combining with the previous observation that predictions with higher frequencies match the benchmark better, we conclude that our strategy of ranking predictions according to their frequencies is reasonable.

\section{Effects of parameters in phase 1}

There are several parameters associated with the proposed GFA algorithm. It is therefore important to see how these parameters affect the performance of GFA. Here we discuss the influence of two parameters, MaxSize and MaxIter, in phase 1, because these two parameters are more important than the parameters in phase 2 . Since phase 1 is time consuming, we are only able to consider a limited combinations of these two parameters.

On MIPS-PPI, we compare two configurations. Configuration 1: MaxSize $=20$ and MaxIter $=30$ (default parameters). Configuration 2: MaxSize $=30$ and MaxIter $=20$. The (default) simple strategy is used in phase 2 to combine candidate subgraphs.

On DIP-PPI, we compare two configurations. Configuration 1: MaxSize $=30$ and MaxIter $=30$ (default parameters). Configuration 2: MaxSize $=40$ and MaxIter $=30$. The (default) simple strategy is used in phase 2 to combine candidate subgraphs. 


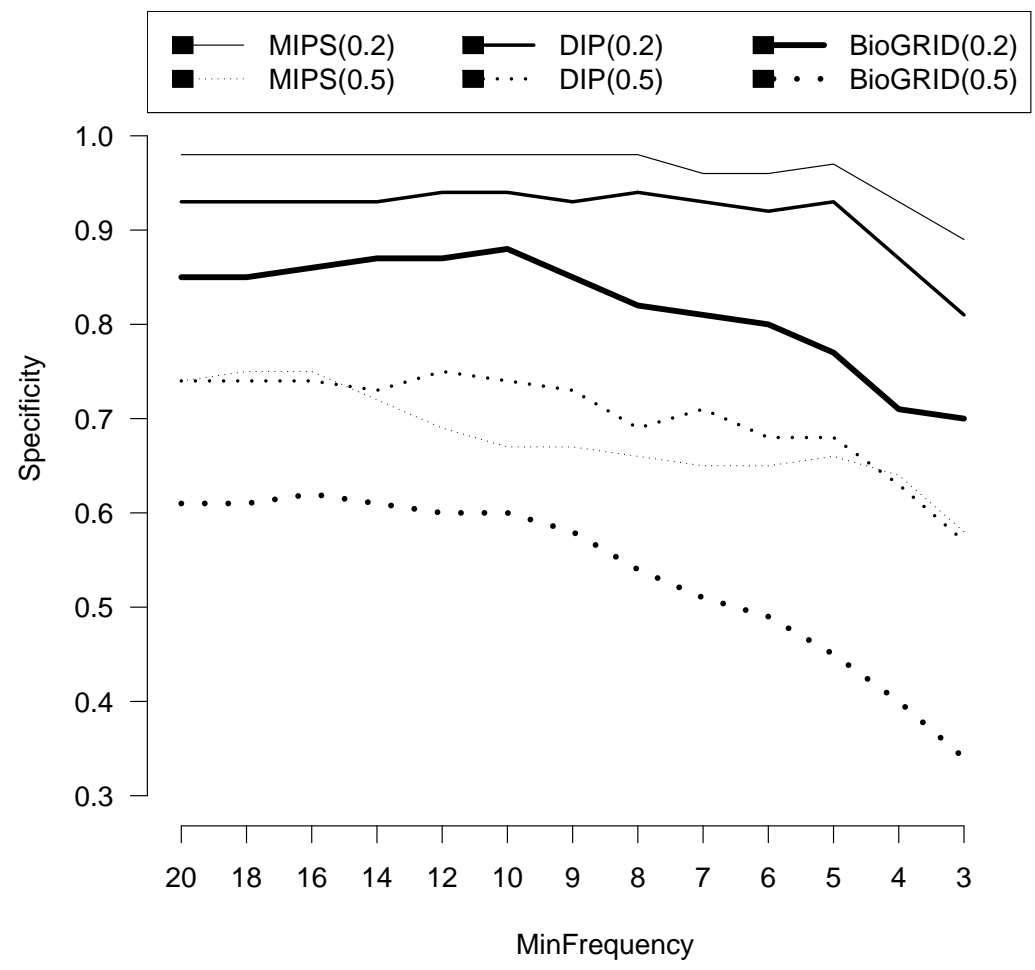

Fig. 4. Comparison of specificities of predictions by GFA when different MinFrequency is used. The cutoffs for the identification are $0.2(\operatorname{MIPS}(0.2), \operatorname{DIP}(0.2)$ and $\operatorname{BioGRID}(0.2))$ and $0.5(\operatorname{MIPS}(0.5), \operatorname{DIP}(0.5)$ and $\operatorname{BioGRID}(0.5))$.

On BioGRID-PPI, we compare three configurations. Configuration 1: Default parameters MaxSize $=40$ and MaxIter $=30$ are used in phase 1 , and the combined strategy is used in phase 2. Configuration 2: Default parameters MaxSize $=40$ and MaxIter $=30$ are used in phase 1, and the simple strategy is used in phase 2. Configuration 3: MaxSize $=50$ and MaxIter $=20$ are used in phase 1, and the combined strategy is used in phase 2. Different strategies for combining candidate subgraphs are considered here to evaluate their influences.

The overall performance of GFA under different configurations are shown in Table V, where MinFrequency is fixed as 3. From the table, we can see that both the sensitivity and specificity vary with different configurations. The differences among them in different configurations are, however, very small. In other words, the comparison shows that the performance of GFA is not very sensitive to MaxSize or MaxIter. 


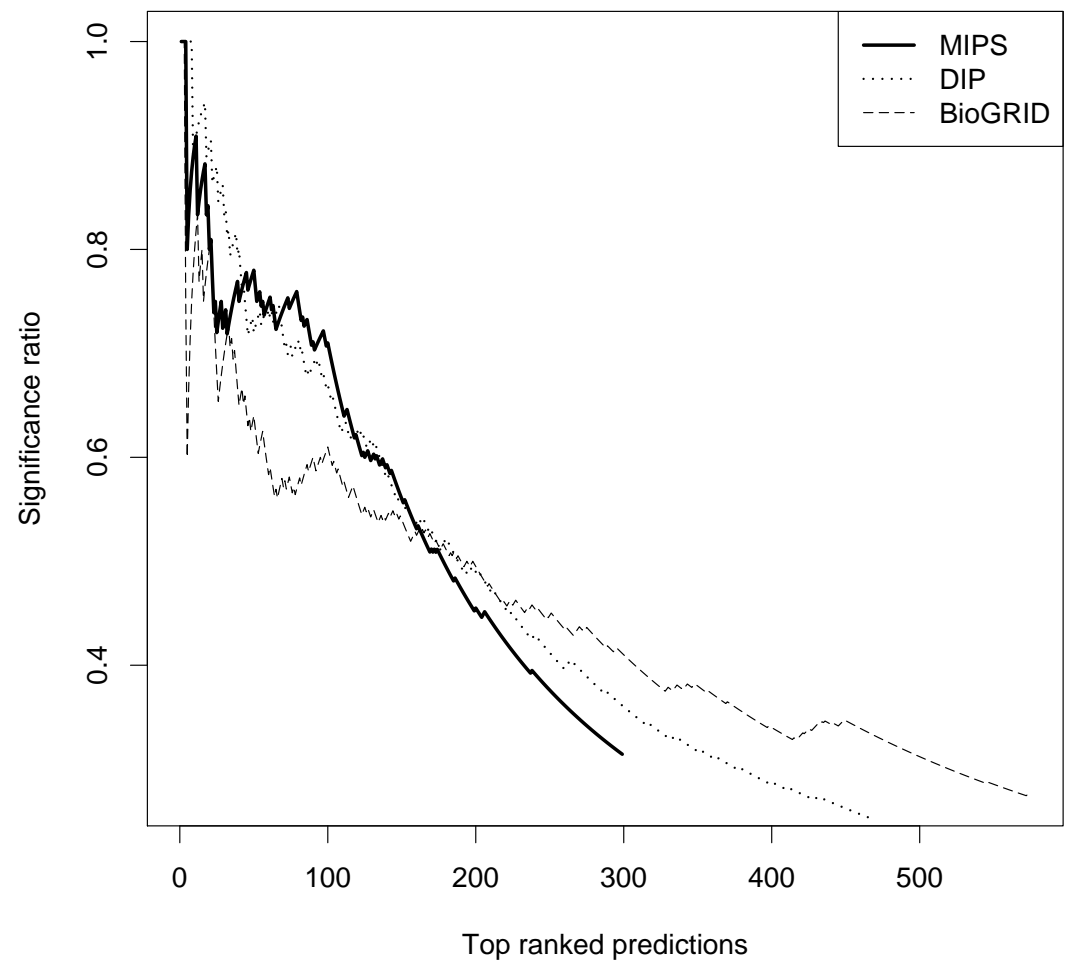

Fig. 5. The significance ratio of top ranked predictions by GFA. The (corrected) p-value threshold for significance is 0.05 .

TABLE V

SENSITIVITY AND SPECIFICITY IN DIFFERENT

CONFIGURATIONS

\begin{tabular}{lccc}
\hline & MIPS-PPI & DIP-PPI & BioGRID-PPI \\
\hline Configuration 1 & $(0.68,0.89)$ & $(0.71,0.81)$ & $(0.54,0.70)$ \\
Configuration 2 & $(0.67,0.89)$ & $(0.69,0.88)$ & $(0.49,0.68)$ \\
Configuration 3 & $\backslash$ & $\backslash$ & $(0.53,0.67)$ \\
\hline
\end{tabular}

MinFrequency is fixed to 3 . The (sensitivity, specificity) pair in each cell is calculated with the identification cutoff being $p=0.2$.

\section{E. Effects of microarray sample size}

In section III-B, we have shown that microarray data do help the identification of protein complexes. It remains interesting to see how the number of samples in the microarray data affect 
the prediction results. For this purpose, we compare the performance of GFA with different number of samples, and show the results for the various configurations defined in the last subsection on BioGRID-PPI in Figure 6
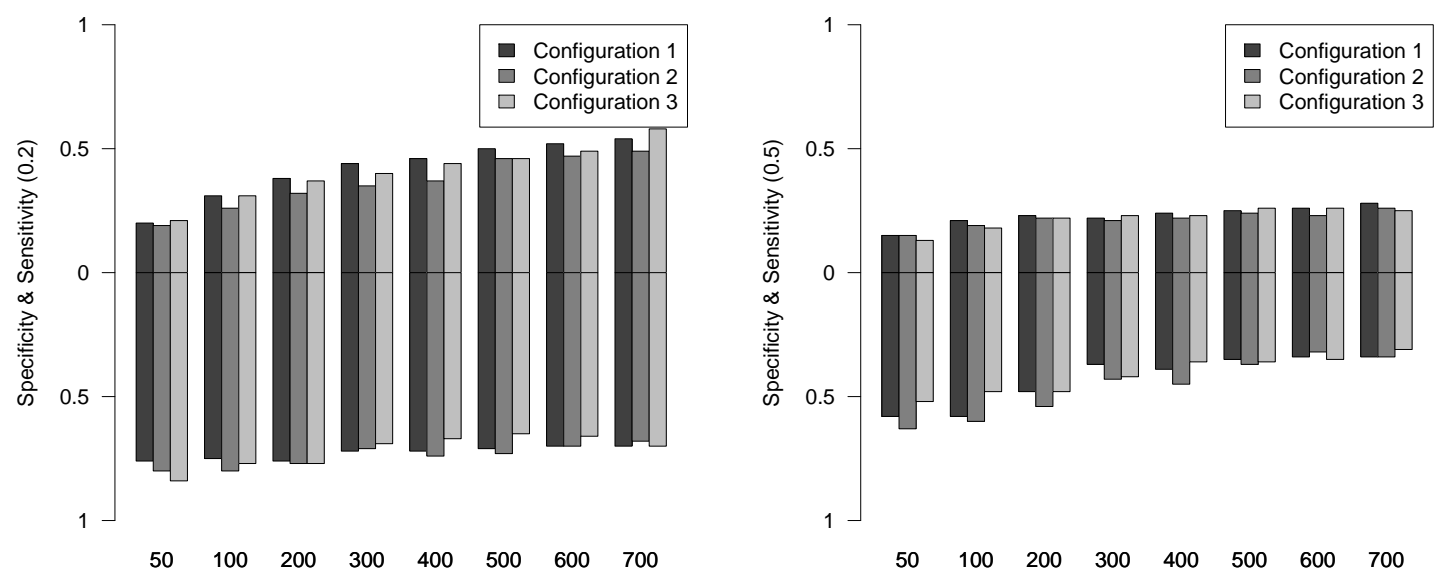

Fig. 6. The effect of sample size on BioGRID-PPI. The cutoffs for the identification are $p=0.2$ (left) and $p=0.5$ (right).

These figures show that on BioGRID-PPI, the sensitivity of GFA increases as the number of samples increases, although the increase slows down after 500 samples. This is because as more samples are used, more complexes are predicted with each frequency. For the same reason and a fixed MinFrequency, the specificity of GFA decreases. To maintain the same specificity, a larger MinFrequency should be selected when more samples are used. The comparison between Configuration 1 and Configuration 2 shows that the combined strategy in phase 2 provides a better sensitivity with some sacrifice in specificity when the number of samples is insufficient. This observation is useful when the PPI network studied is very dense. Because a dense PPI network likely contains many large dense subgraphs, a large number of samples are required in order for GFA to recover these dense subgraphs with sufficient frequency counts. On sparse or moderately-dense PPI networks such as MIPS-PPI or DIP-PPI, the simple strategy of combining candidate subgraphs (and counting frequency) suffices when a few hundred samples are applied. 


\section{Conclusions And Discussion}

We have presented a max-flow based algorithm, GFA, to identify complexes from PPI networks by incorporating microarray data. Compared to the previous methods, GFA is actually able to find the densest subgraphs in the input PPI network efficiently, rather than using some local search heuristic. Our experiments on the MIPS, DIP and BioGRID PPI networks have demonstrated that GFA outperforms the previous methods for predicting protein complexes in terms of specificity while maintaining a comparable sensitivity. Its predicted complexes are highly efficient because they do not overlap much with each other. One of the reasons that GFA was not able to identify some of the benchmark protein complexes is that it removed nodes of degree 1 from the network in every iteration. This step is necessary since it prevents GFA from producing many small spurious predictions. We may have to explore a different strategy in order to improve its sensitivity.

In phase 1 of GFA, multiple rounds of DSA have to be executed in order to find a dense subgraph of a sufficiently small size. This is time consuming. To speed up this step, we can set a small MaxIter. We have demonstrated that the final result is not very sensitive to this parameter. An alternative is to assign larger weights to nodes based on expression data in each round.

As mentioned before, GFA uses the multiplying factor $e^{-\operatorname{expr}(A)}$ to reduce the weights of highly expressed genes in order to fragment a large dense subgraph. This favors highly expressed genes and might tend to ignore some of the genes whose expression levels are significantly lower than their normal levels. A possible solution is to rerun GFA with the reciprocal multiplying factor $e^{\operatorname{expr}(A)}$ to favor lowly expressed genes and combine the predicted complexes appropriately with those obtained with the original multiplying factor $e^{-\exp (A)}$. We have done some preliminary testing on this strategy and found that it slightly improved GFA's performance on some datasets. We will investigate the strategy as well as the issue of optimizing the multiplying factor more carefully in future work.

Our discussion in the previous section shows that the performance of GFA generally improves when more samples are combined. However, the running time of GFA is proportional to the number of samples and could become a concern when the PPI network is large/dense. For example, in our tests, on MIPS-PPI (500 samples), DIP-PPI (600 samples) and BioGRID-PPI (700 samples), GFA needs about 4, 15, and 96 hours (the exact running time depends on the 
parameters used), respectively, on a standard desktop PC. When compared with MCL, MCODE, and MATISSE, which cost less than one hour on BioGRID-PPI, GFA is much slower. On the other hand, since phase 1 of GFA (which costs the most time) can be executed independently on each microarray sample, it is quite straightforward to run GFA in parallel on a computer cluster to achieve a reasonable speed.

\section{ACKNOWLEDGMENTS}

We would like thank the anonymous referees for their many valuable suggestions and comments. This work was partly supported by the Natural Science Foundation of China grants 60805010, 60621062, 60503001, 60528001, and 60575014, the Hi-Tech Research and Development Program of China (863 project) grants 2006AA01Z102 and 2006AA02Z325, NSF grant IIS0711129, NIH grant LM008991, Changjiang and Bioinformatics Chair Visiting Professorships at Tsinghua University.

\section{REFERENCES}

[1] P. Uetz, L. Giot, G. Cagney, T. A. Mansfield, R. S. Judson, J. R. Knight, D. Lockshon, V. Narayan, M. Srinivasan, P. Pochart, A. Qureshi Emili, Y. Li, B. Godwin, D. Conover, T. Kalbfleisch, G. Vijayadamodar, M. Yang, M. Johnston, S. Fields, and J. M. Rothberg, "A comprehensive analysis of protein-protein interactions in saccharomyces cerevisiae," Nature, vol. 403, no. 6770, pp. 623-627, 2000.

[2] T. Ito, K. Tashiro, S. Muta, R. Ozawa, T. Chiba, M. Nishizawa, K. Yamamoto, S. Kuhara, and Y. Sakaki, "Toward a protein-protein interaction map of the budding yeast: a. comprehensive system to examine two-hybrid interactions in all possible combinations between the yeast proteins," Proc. Natl Acad. Sci. USA, vol. 97, no. 3, pp. 1143-1147, 2000.

[3] T. Ito, T. Chiba, R. Ozawa, M. Yoshida, M. Hattori, and Y. Sakaki, "A comprehensive two-hybrid analysis to explore the yeast protein interactome," Proc. Natl Acad. Sci. USA, vol. 98, no. 8, pp. 4569-4574, 2001.

[4] Y. Ho, A. Gruhler, A. Heilbut, G. D. Bader, L. Moore, S.-L. Adams, A. Millar, P. Taylor, K. Bennett, K. Boutilier, L. Yang, C. Wolting, I. Donaldson, S. Schandorff, J. Shewnarane, M. Vo, J. Taggart, M. Goudreault, B. Muskat, C. Alfarano, D. Dewar, Z. Lin, K. Michalickova, A. R. Willems, H. Sassi, P. A. Nielsen, K. J. Rasmussen, J. R. Andersen, L. E. Johansen, L. H. Hansen, H. Jespersen, A. Podtelejnikov, E. Nielsen, J. Crawford, V. Poulsen, B. D. Sørensen, J. Matthiesen, R. C. Hendrickson, F. Gleeson, T. Pawson, M. F. Moran, D. Durocher, M. Mann, C. W. V. Hogue, D. Figeys, and M. Tyers, "Systematic identification of protein complexes in saccharomyces cerevisiae by mass spectrometry," Nature, vol. 415, no. 6868, pp. 180-183, 2002.

[5] A. C. Gavin, M. Bösche, R. Krause, P. Grandi, M. Marzioch, A. Bauer, J. Schultz, J. M. Rick, A. M. Michon, C. M. Cruciat, M. Remor, C. Höfert, M. Schelder, M. Brajenovic, H. Ruffner, A. Merino, K. Klein, M. Hudak, D. Dickson, T. Rudi, V. Gnau, A. Bauch, S. Bastuck, B. Huhse, C. Leutwein, M. A. Heurtier, R. R. Copley, A. Edelmann, E. Querfurth, V. Rybin, G. Drewes, M. Raida, T. Bouwmeester, P. Bork, B. Seraphin, B. Kuster, G. Neubauer, and G. Superti Furga, "Functional organization of the yeast proteome by systematic analysis of protein complexes," Nature, vol. 415, no. 6868, pp. 141-147, 2002 . 
[6] L. Salwinski, C. S. Miller, A. J. Smith, F. K. Pettit, J. U. Bowie, and D. Eisenberg, "The database of interacting proteins: 2004 update," Nucleic Acids Research, vol. 32, no. Database issue, pp. D449-D451, 2004.

[7] U. Güldener, M. Münsterkötter, G. Kastenmüller, N. Strack, J. v. Helden, C. Lemer, J. Richelles, S. J. Wodak, J. GarcíaMartínez, J. E. Pérez-Ortín, H. Michael, A. Kaps, E. Talla, B. Dujon, B. André, J. L. Souciet, J. D. Montigny, E. Bon, C. Gaillardin, and H. W. Mewes, "CYGD: the comprehensive yeast genome database," Nucleic Acids Research, vol. 33, no. Database issue, pp. D364-D368, 2005.

[8] C. Stark, B. J. Breitkreutz, T. Reguly, L. Boucher, A. Breitkreutz, and M. Tyers, "BioGRID: a. general repository for interaction datasets," Nucleic Acids Research, vol. 34, no. Database issue, pp. D535-D539, 2006.

[9] U. Stelzl, U. Worm, M. Lalowski, C. Haenig, F. H. Brembeck, H. Goehler, M. Stroedicke, M. Zenkner, A. Schoenherr, S. Koeppen, J. Timm, S. Mintzlaff, C. Abraham, N. Bock, S. Kietzmann, A. Goedde, E. Toksöz, A. Droege, S. Krobitsch, B. Korn, et al., "A human protein-protein interaction network: A resource for annotating the proteome," Cell, vol. 122, no. 6, pp. 957-968, 2005.

[10] T. Barrett, D. B. Troup, S. E. Wilhite, P. Ledoux, D. Rudnev, C. Evangelista, I. F. Kim, A. Soboleva, M. Tomashevsky, and R. Edgar, "NCBI GEO: mining tens of millions of expression profiles-database and tools update," Nucleic Acids Research, vol. 35, no. Database issue, pp. D760-D765, 2007.

[11] G. D. Bader and C. W. V. Hogue, "An automated method for finding molecular complexes in large protein interaction networks," BMC Bioinformatics, vol. 4, no. 2, 2003.

[12] V. Spirin and L. A. Mirny, "Protein complexes and functional modules in molecular networks," Proc. Natl Acad. Sci. USA, vol. 100, no. 21, pp. 12 123-12 128, 2003.

[13] P. J. Pei and A. D. Zhang, "A 'seed-refine' algorithm for detecting protein complexes from protein interaction data," IEEE Transactions on Nanobioscience, vol. 6, no. 1, pp. 43-50, 2007.

[14] X. L. Li, S. H. Tan, C. S. Foo, and S. K. Ng, "Interaction graph mining for protein complexes using local clique merging," Genome Informatics, vol. 16, no. 2, pp. 260-269, 2005.

[15] X. L. Li, C. S. Foo, and S. K. Ng, "Discovering protein complexes in dense reliable neighborhoods of protein interaction networks," Comput. Syst. Bioinformatics Conf., vol. 6, pp. 157-168, 2007.

[16] A. H. Y. Tong, B. Drees, G. Nardelli, G. D. Bader, B. Brannetti, L. Castagnoli, M. Evangelista, S. Ferracuti, B. Nelson, S. Paoluzi, M. Quondam, A. Zucconi, C. W. V. Hogue, S. Fields, C. Boone, and G. Cesareni, "A combined experimental and computational strategy to dene protein interaction networks for peptide recognition modules," Science, vol. 295 , no. 5553, pp. 321-324, 2002.

[17] B. Andreopoulos, A. An, X. Wang, M. Faloutsos, and M. Schroeder, "Clustering by common friends finds locally significant proteins mediating modules," Bioinformatics, vol. 23, no. 9, pp. 1124-1131, 2007.

[18] A. D. King, N. Pržulj, and I. Jurisica, "Protein complex prediction via cost-based clustering," Bioinformatics, vol. 20, no. 17, pp. 3013-3020, 2004.

[19] D. Bu, Y. Zhao, L. Cai, H. Xue, X. Zhu, H. Lu, J. Zhang, S. Sun, L. Ling, N. Zhang, G. Li, and R. Chen, “Topological structure analysis of the protein-protein interaction network in budding yeast," Nucleic Acids Research, vol. 31, no. 9, pp. 2443-2450, 2003.

[20] C. Wang, C. Ding, Q. Yang, and S. R. Holbrook, "Consistent dissection of the protein interaction network by combining global and local metrics," Genome Biology, vol. 8, no. 12, p. R271, 2007.

[21] E. Segal, H. Wang, and D. Koller, "Discovering molecular pathways from protein interaction and gene expression data," Bioinformatics, vol. 19, no. Suppl 1, pp. i264-272, 2003. 
[22] J. Chen and B. Yuan, "Detecting functional modules in the yeast protein-protein interaction network," Bioinformatics, vol. 22, no. 18, pp. 2283-2290, 2006.

[23] T. Ideker, O. Ozier, B. Schwikowski, and A. F. Siegel, "Discovering regulatory and signalling circuits in molecular interaction networks," Bioinformatics, vol. 18, no. Suppl 1, pp. S233-S240, 2002.

[24] Z. Guo, Y. Li, X. Gong, C. Yao, W. Ma, D. Wang, Y. Li, J. Zhu, M. Zhang, D. Yang, and J. Wang, "Edge-based scoring and searching method for identifying condition-responsive protein-protein interaction sub-network," Bioinformatics, vol. 23, no. 16, pp. 2121-2128, 2007.

[25] I. Ulitsky and R. Shamir, "Identification of functional modules using network topology and high-throughput data," $B M C$ Syst Biol., vol. 1, no. 8, 2007.

[26] — - "Identifying functional modules using expression profiles and confidence-scored protein interactions," Bioinformatics, vol. 25, no. 9, pp. 1158-1164, 2009.

[27] R. Jansen, D. Greenbaum, and M. Gerstein, "Relating whole-genome expression data with protein-protein interactions," Genome Res, vol. 12, no. 1, pp. 37-46, 2002.

[28] N. Simonis, D. Gonze, C. Orsi, J. v. Helden, and S. J. Wodak, "Modularity of the transcriptional response of protein complexes in yeast," Journal of Molecular Biology, vol. 363, no. 2, pp. 589-610, 2006.

[29] S. Tornow and H. W. Mewes, "Functional modules by relating protein interaction networks and gene expression," Nucleic Acids Research, vol. 31, no. 21, pp. 6283-6289, 2003.

[30] R. Sharan, T. Ideker, B. P. Kelley, R. Shamir, and R. M. Karp, "Identification of protein complexes by comparative analysis of yeast and bacterial protein interaction data," J. Comput. Biol, vol. 12, no. 6, pp. 835-846, 2005.

[31] E. Hirsh and R. Sharan, "Identification of conserved protein complexes based on a model of protein network evolution," Bioinformatics, vol. 23, no. 2, pp. e170-e176, 2007.

[32] J. Flannick, A. Novak, B. S. Srinivasan, H. H. McAdams, and S. Batzoglou, "Graemlin: general and robust alignment of multiple large interaction networks." Genome Res, vol. 16, no. 9, pp. 1169-1181, 2006.

[33] R. Sharan and T. Ideker, "Modeling cellular machinery through biological network comparison," Nat Biotech, vol. 24, no. 4, pp. 427-433, 2006.

[34] Z. Li, S. Zhang, Y. Wang, X. S. Zhang, and L. Chen, "Alignment of molecular networks by integer quadratic programming," Bioinformatics, vol. 23, no. 13, pp. 1631-1639, 2007.

[35] G. Gallo, M. D. Grigoriadis, and R. E. Tarjan, "A fast parametric maximum flow algorithm and applications," SIAM J. Comput, vol. 18, no. 1, pp. 30-55, 1989.

[36] S. van Dongen, Ph.D. dissertation, University of Utrecht, 2000.

[37] G. Geva and R. Sharan, " Identification of protein complexes from co-immunoprecipitation data," unpublished manuscript, 2008.

[38] The Gene Ontology Consortium, "Gene ontology: tool for the unification of biology," Nat Genet, vol. 25, no. 1, pp. 25-29, 2000 .

[39] M. R. Garey and D. S. Johnson, Computers and intractability : a guide to the theory of NP-completeness. W. H. Freeman, 1979.

[40] S. Bauer, S. Grossmann, M. Vingron, and P. N. Robinson, "Ontologizer 2.0-a multifunctional tool for GO term enrichment analysis and data exploration," Bioinformatics, vol. 24, no. 14, pp. 1650-1651, 2008.

[41] T. Beissbarth and T. P. Speed, "Gostat: find statistically overrepresented gene ontologies within a group of genes," Bioinformatics, vol. 20, no. 9, pp. 1464-1465, 2004. 
[42] S. Zhong, K. F. Storch, O. Lipan, M. C. J. Kao, C. J. Weitz, and W. H. Wong, "Gosurfer: a graphical interactive tool for comparative analysis of large gene sets in gene ontology space,” Appl. Bioinformatics, vol. 3, no. 4, pp. 261-264, 2004.

[43] R. Shamir, A. Maron Katz, A. Tanay, C. Linhart, I. Steinfeld, R. Sharan, Y. Shiloh, and R. Elkon, "Expander-an integrative program suite for microarray data analysis.” BMC Bioinformatics, vol. 6, no. 232, 2005.

[44] R. G. Miller, Simultaneous statistical inference, 2nd ed. Springer Verlag, 1981.

[45] D. Kressler, P. Linder, and J. d. 1. Cruz, "Protein trans-acting factors involved in ribosome biogenesis in saccharomyces cerevisiae," Mol Cell Biol., vol. 19, no. 12, pp. 7897-7912, 1999.

[46] S. Brohée and J. v. Helden, "Evaluation of clustering algorithms for protein-protein interaction networks," $B M C$ Bioinformatics, vol. 7, no. 488, 2006.

[47] T. Ideker, V. Thorsson, A. F. Siegel, and L. E. Hood, "Testing for differentially-expressed genes by maximum-likelihood analysis of microarray data," Journal of Computational Biology, vol. 7, no. 6, pp. 805-817, 2000.

[48] S. R. Collins, P. Kemmeren, X.-C. Zhao, J. F. Greenblatt, F. Spencer, F. C. P. Holstege, J. S. Weissman, and N. J. Krogan, "Toward a Comprehensive Atlas of the Physical Interactome of Saccharomyces cerevisiae," Mol Cell Proteomics, vol. 6, no. 3, pp. 439-450, 2007.

[49] A. P. Gasch, M. Huang, S. Metzner, D. Botstein, S. J. Elledge, and P. O. Brown, "Genomic Expression Responses to DNA-damaging Agents and the Regulatory Role of the Yeast ATR Homolog Mec1p," Mol. Biol. Cell, vol. 12, no. 10, pp. 2987-3003, 2001. 